\title{
Neuroepidemiologic and Neurobehavioral Characteristics of Motoric Cognitive Risk Syndrome in an Old-Old Population: The Kurihara Project
}

\author{
Keiichi Kumai Kenichi Meguro Mari Kasai Kei Nakamura \\ Masahiro Nakatsuka the Kurihara Project members \\ Division of Geriatric Behavioral Neurology, Cyclotron and Radioisotope Center (CYRIC), \\ Tohoku University, Sendai, Japan
}

Key Words

Motoric cognitive risk syndrome · Gait · Executive function

\begin{abstract}
Background: Recently, the concept of motoric cognitive risk (MCR) syndrome was proposed, where slow gait is considered a risk factor for conversion to dementia. Aim: To retrospectively investigate the characteristics of MCR among a population aged 75+ years, focusing on the aspects of epidemiology and neurobehavioral characteristics. Method: The participants were 590 residents aged 75+ years living in Kurihara who underwent MRI and neurobehavioral assessments including walking velocity. The prevalence of MCR and conversion to dementia (AD8 Dementia Screening Interview cutoff 2/8), together with the neurobehavioral characteristics of the MCR group, were analyzed. Results: The prevalence was $11.1 \%$, and the conversion ratio in the MCR group was higher than that in the non-MCR group (OR $=1.38$ ). The MCR group had lower scores on the executive function test as well as gait velocity. Conclusions: The MCR syndrome increases the rate of conversion to dementia, and both slow gait and lower scores in executive tests, which are 'frontal-based' functions, are predictive of higher rates of conversion to dementia.

(C) 2016 The Author(s)

Published by S. Karger AG, Basel
\end{abstract}

\section{Introduction}

The detection of older residents in the community with mild cognitive impairment that convert to dementia is important when establishing health policies to provide early intervention and prevent further decline to dementia. Neuropsychological tests administered by 
Kumai et al.: Neuroepidemiologic and Neurobehavioral Characteristics of Motoric Cognitive Risk Syndrome in an Old-Old Population: The Kurihara Project

testers much younger than these older residents may lead to a stressful psychological burden, and the impact of lower educational levels on the test results [1] may also make interpretation difficult. One alternative is the use of an observational method such as Clinical Dementia Rating (CDR) [2, 3]. However, the method requires reliable collateral information from the families, and the recent social situation of an increasing number of older adults living alone could prevent the obtainment of reliable information.

Recently, Verghese et al. [4,5] proposed the concept of motoric cognitive risk (MCR) syndrome, which is composed of: (1) cognitive complaints; (2) slow gait, defined as speed 1 SD or more below the age- and sex-matched appropriate mean values in the same cohort; (3) preserved activities of daily life, and (4) absence of dementia. They maintained that older residents whose test scores might be affected by low education could be assessed by the simple method of walking; people who cannot culturally accept neuropsychological tests, or people living alone, are considered for such an evaluation. They found that the people with MCR syndrome showed a higher rate of conversion to dementia.

The neuroepidemiologic (prevalence and rate of conversion to dementia) and neurobehavioral characteristics of MCR syndrome among older adults should be confirmed; however, there has been very limited research with respect to this kind of integrative viewpoint.

We previously performed a community-based study on stroke, dementia, and prevention of confinement to bed in an old-old population aged 75+ years living in the city of Kurihara, Miyagi Prefecture, northern Japan [6,7]. This is a typical agricultural area with a total population of 78,773, and there were 1,254 individuals aged 75+ years in 2008.

The purpose of the current study was to investigate the characteristics of MCR syndrome in this population aged 75+ years using the retrospective database of the Kurihara Project, particularly focusing on (1) neuroepidemiology, i.e., prevalence and rate of conversion to dementia, and (2) neurobehavioral characteristics. This is the first neuroepidemiologic and neurobehavioral study on MCR syndrome in Japan.

\section{Subjects and Methods}

\section{Study Design}

Figure 1 presents the study design. The methodology has been described previously [6]. Briefly, 590 participants from among the target population of 1,254 individuals (47\% response rate) underwent CDR assessment, neurobehavioral testing, and MRI scanning. Of the 590 participants, 223, 293, and 74 were diagnosed as CDR 0 (healthy), CDR 0.5 (questionable dementia), and CDR 1+ (dementia), respectively. The subjects with MCR syndrome (no dementia) were considered to be among the CDR 0 and 0.5 population; thus, $516(223+293)$ people were targeted for the cross-sectional study (see sections Neuroepidemiologic Features and Neurobehavioral Characteristics). For the follow-up study, 474 people were examined.

\section{Criteria for MCR Syndrome}

With reference to the criteria for MCR syndrome proposed by Verghese et al. [4, 5], the diagnostic criteria were retrofit for this study, as follows:

1 Cognitive complaints: positive response to 1 item from the Geriatric Depression Scale (GDS)-15 (more memory problems than most) [8]

2 Slow gait: the maximum gait speed was 1 SD below the mean of those without dementia and disability of activities of daily living (ADL) (CDR 0 or 0.5 )

3 Preserved ADL: intact personal care in the total CDR

4 Absence of dementia: DSM-IV (done by doctors) 
Fig. 1. A total of 590 residents from among the 1,254 people aged $75+$ years living in Kurihara participated in the study and underwent MRI and neurobehavioral assessments, including walking velocity. Possible subjects with MCR syndrome were in the CDR 0 or 0.5 groups; 474 people with CDR 0 or 0.5 were followed up for 3-5 years.
(C) 2016 The Author(s). Published by S. Karger AG, Basel www.karger.com/dee

Kumai et al.: Neuroepidemiologic and Neurobehavioral Characteristics of Motoric Cognitive Risk Syndrome in an Old-Old Population: The Kurihara Project

\begin{tabular}{|c|c|c|c|}
\hline \multirow{4}{*}{$\begin{array}{l}\text { Prevalence } \\
\text { study } \\
2008\end{array}$} & \multicolumn{3}{|c|}{1,254 people aged $75+$ years living in Kurihara } \\
\hline & \multicolumn{3}{|c|}{$\begin{array}{l}590 \text { residents participated } \\
\text { MRI and neurobehavioral assessments }\end{array}$} \\
\hline & $\begin{array}{l}\text { CDR } 0 \text { and } 0.5 \\
\quad n=516\end{array}$ & \multirow[t]{2}{*}{$\begin{array}{c}\text { MCR } \\
\text { syndrome }\end{array}$} & $\begin{array}{c}\text { CDR 1+ } \\
\text { (dementia) } n=74\end{array}$ \\
\hline & Follow-up: $n=474$ & & \\
\hline \multirow{2}{*}{$\begin{array}{l}\text { Incidence } \\
\text { study } \\
2013\end{array}$} & $3-5$ years & & \\
\hline & \multicolumn{2}{|c|}{ Dementia? } & \\
\hline
\end{tabular}

\section{Measures}

Cognitive Function

General cognitive function was assessed using the Mini-Mental State Examination (MMSE) [9], and executive function was evaluated using the Trail Making Test (TMT)-A and the digit symbol substitution test. We considered that executive function was associated with MCR syndrome; thus, no intensive memory tests (e.g., the full version of the Wechsler Memory Scale-Revised) were performed.

\section{Gait Velocity}

According to the protocol of the Kurihara Project, we asked the participants to walk as fast as possible for $6 \mathrm{~m}$. We asked the participants to walk 2 times, and the mean value was used for the analyses.

\section{Analyses}

Neuroepidemiologic Features

The prevalence rate of MCR syndrome was calculated retrospectively from the database of the Kurihara Project. Regarding the rate of conversion to dementia, unfortunately the situation in the city prevented us from performing an incidence study on the same cohort in Kurihara. Thus, we decided to send the AD8 Dementia Screening Interview (AD8) $[10,11]$ questionnaire to the participants in the prevalence study by mail. The cutoff for the AD8 was $2 / 8[11]$.

Since the time interval between the prevalence study and follow-up with the AD8 questionnaire ranged from 3 to 5 years, we applied the person-year method when calculating the rate of conversion to dementia. Since the CDR is an established method of classifying people into healthy subjects (CDR 0) and those with a borderline condition (CDR 0.5), with the latter including converters to dementia, a CDR-versus-MCR group analysis was also performed to assess converters to dementia.

\section{Neurobehavioral Characteristics}

After classifying the participants into two groups, i.e., an MCR and a non-MCR group, neurobehavioral differences were analyzed. For executive function, one-way ANCOVA with the covariate of MMSE was used.

Neurological Background

When evaluating the neurological background of the MCR syndrome, the subjects were classified into four groups at baseline (prevalence data), i.e., CDR 0, CDR 0.5-very mild Alzheimer's disease (AD), CDR 0.5-very mild subcortical vascular dementia (SVD), and other CDR 0.5. 
Table 1. Rates of conversion to dementia

\begin{tabular}{lllll} 
& Entry cases & Incidence cases & $\begin{array}{l}\text { Person-years } \\
(3-5 \text { years })\end{array}$ & Incidence rate $/ 1,000, \% 0^{\mathrm{a}}$ \\
\hline MCR group & 35 & 15 & 125.2 & 119.8 \\
Non-MCR group & 264 & 88 & 858.8 & 102.5 \\
\hline
\end{tabular}

Assessment of dementia is based on the AD8. ${ }^{a}$ Incidence rate/1,000 = (number of incidence cases/ person-years) $\times 1,000$.

The very mild AD group met the all of the following criteria: (1) the total CDR was 0.5, with the CDR domain of memory being 0.5 as well; (2) no neurological symptoms; (3) with or without executive dysfunction, and (4) no CVD with or without white matter changes as assessed with FLAIR MRI. The presence of executive dysfunction was defined here as a time 1 SD longer (in seconds) to complete the TMT-A, more than $300 \mathrm{~s}$ to complete the TMT-B, or incomplete results.

Very mild SVD was defined as follows: (1) the total CDR was 0.5, with the CDR domain of memory being 0.5 as well; (2) positive neurological symptoms; (3) presence of executive dysfunction, and (4) presence of CVD or white matter changes as assessed with FLAIR MRI. The criteria were modified from those of Erkinjuntti et al. [12].

\section{Results}

\section{Neuroepidemiologic Features}

Prevalence

The prevalence of MCR syndrome was calculated to be $11.1 \%$ (9.5\% for men, $12.1 \%$ for women) among the old-old population aged $75+$ years of this study. This rate was consistent with the findings of Verghese et al. [4]: among the older adults aged $60+$ years $(n=26,802)$, the prevalence was $9.7 \%$ (95\% CI: 8.2-11.2), and among those aged $75+$ years $(n=8,651)$, the prevalence was $10.6 \%$ (95\% CI: 9.0-12.3).

\section{Rate of Conversion to Dementia}

Effective responses were obtained from 299 participants of the initial population of 474 (63.1\%). Table 1 presents the rates of conversion to dementia for the MCR group versus the non-MCR group. Both groups showed high conversion rates, even though that of the MCR group was higher than that of the non-MCR group.

\section{Neurobehavioral Characteristics}

Table 2 presents the neurobehavioral characteristics of the MCR and non-MCR groups. The MCR group had a lower level of education, lower scores on the executive function test (TMT-A and digit symbol substitution test), and reduced gait velocity.

\section{Neurological Background}

Table 3 presents the incidence rates of MCR versus non-MCR syndromes for the four groups (CDR 0 , very mild AD, very mild SVD, and other CDR 0.5 ). No differences were evident $\left(\chi^{2}\right.$ test $)$. 
Dementia

and Geriatric

Table 2. Neurobehavioral characteristics

Table 3. Neurological background

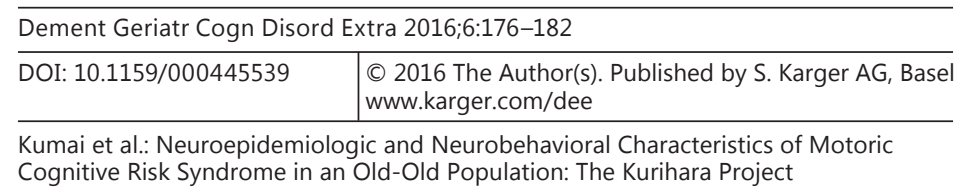

\begin{tabular}{lccc}
\hline & MCR group & Non-MCR group & p value \\
\hline Subjects (men/women) & $57(19 / 38)$ & $456(181 / 275)$ & \\
Age, years & $80.4(4.3)$ & $79.7(4.0)$ & 0.25 \\
Education, years & $8.0(1.0)$ & $9.0(2.0)$ & $<0.001$ \\
MMSE score & $22.8(3.5)$ & $24.1(3.3)$ & 0.01 \\
TMT-A score & $92.6(34.8)$ & $74.7(28.8)$ & $<0.001^{*}$ \\
Digit symbol 120 s score & $25.9(9.3)$ & $31.8(11.5)$ & $<0.001^{*}$ \\
Gait velocity, m/s & $0.8(0.1)$ & $1.2(0.3)$ & $<0.001$ \\
\hline
\end{tabular}

* Significant by one-way ANCOVA with the covariate of MMSE.

\begin{tabular}{lllll}
\hline \multirow{2}{*}{ CDR 0 } & \multicolumn{2}{l}{ CDR 0.5 } & \\
\cline { 3 - 5 } & & very mild AD & very mild SVD other CDR 0.5 \\
\hline MCR group & $2 / 9$ & $5 / 8$ & $2 / 3$ & $6 / 15$ \\
Non-MCR group & $31 / 129$ & $17 / 44$ & $12 / 26$ & $28 / 65$ \\
\hline
\end{tabular}

Incidence rate/total baseline number in the incidence study of 2013.

\section{Discussion}

\section{Methodological Issues}

Before discussing the results, there are some methodological issues to note.

\section{MCR Criteria}

The original criteria by Verghese et al. [4, 5] involved the following: (1) 'cognitive complaints' utilized the CERAD, the Albert Einstein Health Self-Assessment Form, and 1 item from the GDS-15; (2) 'slow gait' took into consideration the normal gait speed as assessed with the mean \pm 1 SD without dementia or disability of ADL; (3) 'preserved ADL' involved no disability (inability or requiring assistance in performing ADL), and (4) 'absence of dementia' was based on the DSM-IV. Because the Kurihara Project database was utilized, we had no choice but to use maximum speed for criterion 2. While this was different from the original criterion, we considered that maximum gait speed would be better than normal gait speed to elucidate the difference between healthy aging and MCR syndrome. Healthy older adults may walk at a slow pace; however, they are able to walk at maximum speed when requested.

Dementia Incidence

As described earlier, we had to use the AD8 questionnaire instead of conducting an incidence study. The 299 participants of the original 1,200+ subjects considered for the incidence analysis might supposedly have introduced a selection bias. Furthermore, we applied the person-year method for calculating the rate of conversion to dementia, since the interval between the prevalence study and the follow-up questionnaire ranged from 3 to 5 years. The AD8 has been reported as a good indicator of mild cognitive impairment and dementia. Thus, we considered that the results would provide useful information as to the risk of dementia in MCR subjects. 
Kumai et al.: Neuroepidemiologic and Neurobehavioral Characteristics of Motoric Cognitive Risk Syndrome in an Old-Old Population: The Kurihara Project

\section{Neuroepidemiology}

The prevalence of MCR syndrome calculated herein was consistent with the findings of Verghese et al. [4, 5], despite the difference in diagnostic criteria. The results regarding incidence rates were also consistent with the previous findings. The fact that these measures were similar supports the validity of the clinical diagnosis of MCR syndrome.

Neurobehavioral Characteristics: Not General Cognitive Function but Executive Function

What we describe as 'general cognitive function', as frequently assessed with the MMSE, is considered not to be 'general' but language-based cognitive function. While the left hemisphere is believed to be utilized, exercise or walking control is based on the frontal part of the brain. In this sense, the finding that similar 'frontal-based' executive function was associated with maximum gait velocity is considered reasonable.

For neuropsychological testing, we usually take the digit symbol substitution test or TMT-A/B as executive function tests, which focus on the upper extremities. We consider maximum gait velocity to represent executive function using the lower extremities; thus, we should explicitly control gait in order to maximize the potential competence level, together with controlling for risks such as falling.

\section{Neurological Background}

The lack of a difference in the distribution of CDR 0 , very mild AD, very mild SVD, and other CDR 0.5 between the MCR and the non-MCR group indicates that MCR syndrome has no specific neuropathological background. Although we did not diagnose the dementing diseases at the outset of administering the AD8 questionnaire, we did not demonstrate any predominance of vascular lesions among the subjects with MCR syndrome. Three reasons are considered. Firstly, since the diagnostic criterion of Verghese et al. [4, 5] for vascular dementia was the DSM-IV, which may include AD with CVD among vascular dementia, overdiagnosis might have occurred [13]. Secondly, subjects with very mild AD also exhibited executive dysfunction, which met the criterion for MCR syndrome. Thirdly, subjects with very mild SVD easily manifest apathy and might withdraw from the community [14]. Further investigation is necessary to elucidate these points.

\section{Conclusions}

As described earlier, neuropsychological testing may lead to a stressful psychological burden, and the impact of lower educational level on test results may also make their interpretation difficult. An observational method requires reliable collateral information from the families, and the recent social situation of an increasing number of older adults living alone could prevent the obtainment of reliable information. Our findings suggest that the concept of MCR syndrome has clinical utility, since the syndrome increases the rate of conversion to dementia. Both slow gait and lower scores in executive tests, which are 'frontal-based' functions, are predictive of higher rates of conversion to dementia.

\section{Disclosure Statement}

No disclosures. 
Kumai et al.: Neuroepidemiologic and Neurobehavioral Characteristics of Motoric Cognitive Risk Syndrome in an Old-Old Population: The Kurihara Project

\section{References}

1 Meguro K, Shimada M, Yamaguchi S, Ishizaki J, Ishii H, Shimada Y, Sato M, Yamadori A, Sekita Y: Cognitive function and frontal lobe atrophy in normal elderly adults: implications for dementia not as aging-related disorders and the reserve hypothesis. Psychiatry Clin Neurosci 2001;55:565-572.

-2 Morris JC: The Clinical Dementia Rating (CDR): current version and scoring rules. Neurology 1993;43:24122414.

3 Meguro K: A Clinical Approach to Dementia: An Instruction of CDR Worksheet (in Japanese). Tokyo, Igakushoin, 2004.

4 Verghese J, Annweiler C, Ayers E, Barzilai N, Beauchet O, Bennett DA, Bridenbaugh SA, Buchman AS, Callisaya ML, Camicioli R, Capistrant B, Chatterji S, De Cock AM, Ferrucci L, Giladi N, Guralnik JM, Hausdorff JM, Holtzer R, Kim KW, Kowal P, Kressig RW, Lim JY, Lord S, Meguro K, Montero-Odasso M, Muir-Hunter SW, Noone ML, Rochester L, Srikanth V, Wang C: Motoric cognitive risk syndrome: multicountry prevalence and dementia risk. Neurology 2014;83:718-726.

5 Verghese J, Ayers E, Barzilai N, Bennett DA, Buchman AS, Holtzer R, Katz MJ, Lipton RB, Wang C: Motoric cognitive risk syndrome: multicenter incidence study. Neurology 2014;83:2278-2284.

6 Meguro K, Tanaka N, Kasai M, et al: Prevalence of dementia and dementing diseases in the old-old population in Japan: the Kurihara Project. Implications for Long-Term Care Insurance data. Psychogeriatrics 2012;12: 226-234.

7 Nakatsuka M, Nakamura K, Hamanosono R, Takahashi Y, Kasai M, Sato Y, Suto T, Nagatomi R, Meguro K: A cluster randomized controlled trial of nonpharmacological interventions for old-old subjects with a Clinical Dementia Rating of 0.5: the Kurihara Project. Dement Geriatr Cogn Dis Extra 2015;5:221-232.

-8 Sutcliffe C, Cordingley L, Burns A, Mozley CG, Bagley H, Huxley P, Challis D: A new version of the Geriatric Depression Scale for nursing and residential home populations: the Geriatric Depression Scale (Residential) (GDS-12R). Int Psychogeriatr 2000;12:173-181.

-9 Folstein MF, Folstein SE, McHugh PR: 'Mini-mental state'. A practical method for grading the cognitive state of patients for the clinician. J Psychiatr Res 1975;12:189-198.

10 Galvin JE, Roe CM, Powlishta KK, Coats MA, Muich SJ, Grant E, Miller JP, Storandt M, Morris JC: The AD8: a brief informant interview to detect dementia. Neurology 2005;65:559-564.

11 Meguro K, Kasai M, Nakamura K; Kurihara Project Members: Reliability and validity of the Japanese version of the AD8 (in Japanese). Nihon Ronen Igakkai Zasshi 2015;52:61-70.

12 Erkinjuntti T, Inzitari D, Pantoni L, Wallin A, Scheltens P, Rockwood K, Roman GC, Chui H, Desmond DW: Research criteria for subcortical vascular dementia in clinical trials. J Neural Transm Suppl 2000;59:23-30.

13 Meguro K, Ishii H, Yamaguchi S, Ishizaki J, Shimada M, Sato M, Hashimoto R, Shimada Y, Meguro M, Yamadori A, Sekita Y: Prevalence of dementia and dementing diseases in Japan: the Tajiri Project. Arch Neurol 2002;59: 1109-1114.

14 Nakamura K, Kasai M, Ouchi Y, Nakatsuka M, Tanaka N, Kato Y, Nakai M, Meguro K: Apathy is more severe in vascular than amnestic mild cognitive impairment in a community: the Kurihara Project. Psychiatr Clin Neurosci 2013;67:517-525. 